\title{
Enseñanza de la lectoescritura en entornos virtuales de aprendizaje
}

\section{Teaching literacy in virtual learning environments}

1 Pamela Alexandra Jerez Heredia https://orcid.org/0000-0002-2990-2732 Universidad Técnica de Ambato, Carrera de Psicopedagogía, Ambato, Ecuador. pjerez4233@uta.edu.ec

2 Anthony Marcelo Lara Flores https://orcid.org/0000-0001-6938-0660 Universidad Técnica de Ambato, Carrera de Psicopedagogía, Ambato, Ecuador. alara8704@uta.edu.ec

3 Gissella Alexandra Arroba López (iD) https://orcid.org/0000-0002-7846-6535 Universidad Técnica de Ambato, Carrera de Educación Inicial, Ambato, Ecuador. ga.arroba@uta.edu.ec

4 Ximena Cumandá Miranda López https://orcid.org/0000-0001-6133-1186 Universidad Técnica de Ambato, Carrera de Psicopedagogía, Ambato, Ecuador. xc.miranda@uta.edu.ec

Jerez Heredia, P. A., Lara Flores, A. M., Arroba López, G. A., \& Miranda López, X. C. (2023). Enseñanza de la lectoescritura en entornos virtuales de aprendizaje. ConcienciaDigital, $6(1.4)$, 826-842. https://doi.org/10.33262/concienciadigital.v6i1.4.2031

CONCIENCIA DIGITAL, es una Revista Multidisciplinar, Trimestral, que se publicará en soporte electrónico tiene como misión contribuir a la formación de profesionales competentes con visión humanística y crítica que sean capaces de exponer sus resultados investigativos y científicos en la misma medida que se promueva mediante su intervención cambios positivos en la sociedad. https://concienciadigital.org

La revista es editada por la Editorial Ciencia Digital (Editorial de prestigio registrada en la Cámara Ecuatoriana de Libro con No de Afiliación 663) www.celibro.org.ec 
Palabras claves:

Lectoescritura, enseñanza virtual, lectura, pandemia

Keywords:

Literacy, elearning, reading, pandemic
Resumen

Introducción. Durante la pandemia, fuimos testigos de acontecimientos nunca dados, uno de los más sobresalientes se dio en el contexto de la educación con el traslado del proceso de enseñanza y aprendizaje a ambientes virtuales. La enseñanza de la lectoescritura también es parte de ello por lo que resulta importante analizar cómo se llevó a cabo este proceso durante la emergencia sanitaria, Objetivo. Analizar la repercusión de la enseñanza virtual en el aprendizaje de la lectoescritura Metodología. La investigación es de tipo descriptiva, con un enfoque mixto ya que busca analizar y describir la relación entre la enseñanza virtual y el aprendizaje de la lectoescritura, se aplicó además una modalidad bibliográfica y de campo ya la información fue recolectada de fuentes primarias. Resultados. Los resultados reflejan que un manejo adecuado o inadecuado de las herramientas tecnológicas para la enseñanza de la lectoescritura tiene una fuerte relación con el aprendizaje de competencias de lectura y escritura en los niños de entre 6 y 8 años Conclusión. La aplicación de estrategias metodológicas adaptadas a la nueva modalidad de estudio, inciden de manera significativa en los resultados de aprendizaje de la lectoescritura en niños de entre 6 y 8 años.

\section{Abstract}

Introduction. During the pandemic, we witnessed events never seen before, one of the most outstanding was in the context of education with the transfer of the teaching and learning process to virtual environments. The teaching of literacy is also part of this, so it is important to analyze how this process was carried out during the health emergency, Objective. To analyze the impact of virtual teaching on literacy learning Methodology. The research is descriptive, with a mixed approach since it seeks to analyze and describe the relationship between virtual teaching and learning to read and write, and a bibliographic and field modality was applied since the information was collected from primary sources. Results. The results reflect that an adequate or inadequate use of technological tools for teaching literacy has a strong relationship with the learning of reading and writing skills in children between 6 and 8 years of age Conclusion. The application of methodological strategies adapted to the new study modality has a significant impact on the results of literacy learning in children between 6 and 8 years of age. 


\section{Introducción}

\section{Aprendizaje virtual}

La educación virtual o en línea hace referencia al desarrollo del proceso de enseñanzaaprendizaje mediante espacios virtuales, es decir la interacción entre estudiantes y docentes no se da ni se limita a la presencialidad. Se apoya en las Tecnologías de la Información y Comunicación (TIC) ya que dentro de este proceso se utilizan herramientas que se ofrecen dentro de la red de internet (Aguilar Gordón, 2020).

Esta es una herramienta que en los últimos tiempos ha denotado un auge y alto impacto en la cobertura y la calidad educativa utilizándola en todos los niveles de educación y en los diferentes tipos de formación académica debido a sus rasgos multimediales, hipertextuales e interactivos (Crisol et al., 2020).

\section{Origen}

El aprendizaje o educación virtual se origina con la llegada del internet en la década de los noventa, pero su uso se ha intensificado a partir del año 2020 como una medida de prevención frente al COVID-19; sin embargo, es evidente que no todos los gobiernos, ni los agentes educativos estaban preparados para este cambio de modalidad, ya sea con infraestructura, con conocimientos en cuanto al uso de herramientas tecnológicas y a la práctica metodológica docente, constituyéndose en un verdadero reto educativo (Ramos et al., 2021).

El uso de las Tecnologías de la Información y Comunicación en la actualidad es cada vez mayor y dentro del ámbito educativo también se ha dado de forma progresiva con especial énfasis durante la pandemia por Covid-19. Dentro de este campo se han generado varias innovaciones, nuevas herramientas y competencias que tanto docentes como estudiantes han tenido que desarrollar (Cediilo \& Cevallos, 2020).

\section{Características del aprendizaje virtual}

En la actualidad, los procesos de enseñanza-aprendizaje y la práctica educativa en general, se han fortalecido con el uso de la tecnología. Para lograr resultados eficaces, es imprescindible contar con plataformas tecnológicas estables, que sean factibles, de fácil acceso, que estén al alcance y respondan a las necesidades de los estudiantes (Ramos et al., 2021).

Dentro de este modelo educativo, el rol del docente se basa en diseñar las instrucciones de la clase virtual, proporcionar a los estudiantes material y contenido de aprendizaje basado en e- learning, brindar un feedback que sea efectivo y evaluar el proceso de enseñanzaaprendizaje de manera continua y permanente (Cabezas et al., 2021). 
Por otro lado, el rol del estudiante en esta modalidad de aprendizaje debe fundamentarse en la autodisciplina, en el aprendizaje autónomo y colaborativo, sumando a la construcción de un pensamiento crítico y reflexivo (Norman Acevedo, 2019).

\section{Metodologías en ambientes virtuales}

Las metodologías en ambientes virtuales son un conjunto técnicas, estrategias y métodos que tienen la finalidad fomentar la participación de los estudiantes en la construcción de su propio aprendizaje, generando así aprendizajes más significativos y con profundidad que permitan orientar la adquisición de competencias e impulsar la transferencia de estas (Gómez \& Ostos Ortiz, 2021).

La habilitación de ambientes de aprendizaje virtuales supone cambios en las metodologías de enseñanza y aprendizaje, entre las que destacan: design thinking para la resolución de problemas, Teach back para para el aprendizaje a través de la conversación, Flipped learning para fomentar la independencia y el seguimiento, Gamification para el aprendizaje a través del juego y Social media para impulsar el aprendizaje a través de la red, entre otros (Gómez \& Ostos, 2021).

\section{Competencias digitales docentes}

Existe un amplio campo de competencias digitales que los docentes deben desarrollar, para facilitar su compresión esta se pueden clasificar en diferentes áreas.

\section{Tabla 1}

\section{Competencias digitales docentes}

\begin{tabular}{ll}
\hline \multicolumn{1}{c}{ Áreas } & \multicolumn{1}{c}{ Enunciado } \\
\hline $\begin{array}{l}\text { Información y alfabetización } \\
\text { informacional }\end{array}$ & $\begin{array}{l}\text { Eldocente debe identificar yanalizar información digital } \\
\text { mediante su finalidad y relevancia. }\end{array}$ \\
Comunicación y colaboración & $\begin{array}{l}\text { Permite comunicar y trasmitir la información a través de } \\
\text { herramientas digitales con la colaboración e interacción en red. }\end{array}$ \\
Creación de contenidos digitales & Hace referencia a la capacidad de crear, integrar y reelaborar \\
& $\begin{array}{l}\text { contenidos digitales, y el conocimiento en relación con } \\
\text { derechos de autor y programación. }\end{array}$ \\
Seguridad & Se relaciona con la protección del contenido digital, \\
dispositivos, de datos personales y de la salud y el entorno en & que se desarrolla tanto alumnos como docentes. \\
Utilización de la información y de las herramientas digitales de \\
manera creativa e innovadora e identificación de problemas y \\
sus respuestas tecnológicas.
\end{tabular}




\section{Lectura}

Constituye un proceso dinámico y esencial en el desarrollo cognitivo de los individuos, debido a que tiene una amplia repercusión para la adquisición del conocimiento, la lectura desarrolla funciones metales superiores como el análisis, inferencia y sinterización (Guerrero et al., 2020).

Consiste en la comprensión de un texto u otros medios escritos en donde se decodifica la información, ya sean, gráficos, signos o variedad de estímulos que nuestra mente pueda ser capaz de analizar e interpretar, siendo además una estrategia básica y esencial para desarrollar también habilidades psicológicas y afectivas (Martínez, 2021).

\section{Factores que intervienen en la lectura}

En el desarrollo de la lectura se evidencian 7 factores principales y básicos que son de carácter fundamental. Es esencial identificar cada uno de ellos, para llevar a cabo el desarrollo crítico, la comprensión y una adecuada interpretación de textos. Estos factores son: los conocimientos previos, capacidad de análisis, motivación, vocabulario, concentración, memoria y las estrategias de comprensión lectora, cada uno de los factores mencionados, son necesarios para estimular y desarrollar activamente los procesos lectores (Pérez, 2017).

\section{Tabla 2}

\section{Factores que intervienen en el aprendizaje de la lectura}

\begin{tabular}{cl}
\hline \multicolumn{1}{c}{ Factores } & \multicolumn{1}{c}{ Enunciado } \\
\hline Los conocimientos previos & Es fundamental conocer los saberes previos para la comprensión \\
& lectora, esto proporciona una base sólida al momento de plasmar \\
& métodos para la enseñanza. \\
La capacidad de análisis & La identificación que muestra el sujeto frente a los textos que se le \\
& presenten. \\
El vocabulario & Cuanto conocimiento general de palabras alberga dentro de la \\
& conciencia del niño. \\
La motivación & La emoción natural a la lectura, este factor se lo puede llegar a \\
& implantar en las personas. \\
La concentración & Dentro de la lectura la concentración juega un papel muy importante, \\
& ya que es un proceso en donde idéntica la información. \\
La memoria & Proceso usado para recordar los contenidos manifestados, los cuales \\
& se mantienen un almacenamiento siendo capaces de evocarlo cuando \\
& sea necesario. \\
Eas estrategias de comprensión & Es el factor determinante para lograr una lectura 100\% Comprensiva \\
lectora & consta de una serie de formas para su correcta comprensión, las cuales \\
& son: localizar ideas, comprender secuencia, predicciones, \\
& inferencias, etc...
\end{tabular}

Fuente: Pérez (2017) 


\section{Escritura}

Es una habilidad lingüística fundamental en el proceso del desarrollo, se le conoce usualmente como segunda lengua, ya que cumple con uno de los roles más básicos y elementales el de comunicar, sin embargo, es una de las habilidades de difícil adquisición, por lo que es necesario conocer el proceso ideal para lograr desarrollar dicha habilidad (Erazo et al., 2020).

Factores que intervienen en la escritura

El desarrollo del individuo está marcado por diversos componentes, una serie de procesos que son de vital importancia para el desarrollo de la escritura, la adquisición de la escritura está comprometida con el desarrollo madurativo y fisiológico del niño, tomando en cuenta varios factores por lo que se infiere que la forma en la que sinteticen las personas la información para el aprendizaje será distinta (Pascual et al., 2018).

\section{Tabla 3}

\section{Factores para el aprendizaje de la escritura}

\begin{tabular}{ll}
\hline \multicolumn{1}{c}{ Factor } & \multicolumn{1}{c}{ Enunciado } \\
\hline Factores cognitivos. & Son de gran influencia para aprender a escribir, en donde llega a influir \\
& en gran escala la memoria, tanto como a corto y largo plazo. La atención \\
& juega un rol de suma importancia también en conjunción con el \\
& pensamiento. Son capaces de dar apertura al proceso lector por \\
& completo. $^{\text {a }}$
\end{tabular}

Factores perceptivos. El desarrollo de los sentidos es esencial para que se dé el proceso mental de codificación de elementos almacenados logrando relacionarlos con objetos del mundo real. ${ }^{\mathrm{b}}$

Factores $\quad$ El nivel de maduración psicomotriz influye en el éxito de la lectura, se psicomotrices. debe a que el desarrollo psicomotor es directamente proporcional al desarrollo familiar de otros factores antes mencionados, un niño de 7 años comprenderá mejor que uno de 4 años, se debe a su desarrolla más maduro.

Factores socio- $\quad$ La lectura y escritura vienen a ser objetos culturales, dado que se logran familiares

a prender en situaciones de convivencia, social- familiar, llegan a tener una gran repercusión dentro del éxito para el desarrollo de la lectura y escritura. $^{\mathrm{d}}$

Fuente: ${ }^{1}$ Huaraca (2020). ${ }^{\mathrm{b}}$ Pascual \& Lozano (2020), ${ }^{\mathrm{c}}$ Rodriguez (2019) \& ${ }^{\mathrm{d}}$ Falcon (2020)

\section{Métodos de iniciación para la lectoescritura}

Son varios los métodos utilizados en la enseñanza de la lectoescritura, cada uno con sus características y aplicación específica. Entre los más conocidos encontramos: método fonético, método silábico, método global, entre otros. Una de las características claves de 
estos métodos es la retroalimentación que se pude obtener a través de la aplicación exclusiva de cada uno de ellos o mediante la combinación de estos, para alcanzar los objetivos propuestos a partir de un aprendizaje adaptado a las necesidades específicas de los estudiantes (García \& Pito, 2019).

Tabla 4

Métodos de enseñanza de la lectoescritura

\begin{tabular}{cl}
\hline Métodos & Característica \\
\hline Método fonético o fónico & Se basa en aprender los sonidos para posteriormente \\
& combinarlos y así paulatinamente lograr la adquisición de la \\
& lectura y escritura.
\end{tabular}

Método silábico

Método de las palabras normales

Método alfabético o deletreo

El Método global
Surge a consecuencia de la falta de consistencia en los métodos presentados con anterioridad, este consiste en aprender vocales, luego silabas y luego palabras.

Fue ampliamente defendido por Juan Amos Comenio, debido a su fácil comprensión, consiste en separar la palabra para obtener una palabra generadora, de esta palabra generadora partirá la formación de otras palabras con la misma base generada.

Es usado para enseñar a escribir y leer a los niños, consiste en apoyarse en la memoria del niño verificando la grafía presentada y las relaciones semánticas entre el sentido fonético y escritura de la palabra.

Consiste en la enseñanza de la palabra completa, dejando de lado el aprendizaje básico con lo que se consigue la falta de regularidad del código, centrándose en unidades netamente con significado.

\section{Fuente: García \& Pito (2019)}

\section{Barreras con la enseñanza virtual de la lectoescritura}

En la actualidad, más de 100 millones de niños se muestran muy por debajo del nivel mínimo de competencia en lectura, esto a consecuencia de la pandemia, debido al cierre que hubo en las escuelas a causa del COVID-19.

Durante el año 2020, la cifra de estudiantes con competencias lectoras estaba disminuyendo; sin embargo, para finales de ese mismo año, las cifras aumentaron un $20 \%$, pasando de 483 millones a 584 millones de estudiantes con carencias lectoras; esto significó una catástrofe general, lo cual representa un impacto negativo en el desarrollo mundial y disminuye el gran avance que se había logrado durante los últimos 20 años 
(Sotomayor, 2021).

La modalidad virtual presentó algunas ventajas para la enseñanza gracias al uso de las TIC's; sin embargo, a medida que las semanas y meses transcurrían, las barreras existentes en esta modalidad se hicieron más claras. Barreras como la escasa accesibilidad de algunos estudiantes, la brecha digital existente y las desigualdades sociales, evidenciadas con mayor proporción en los países de Latinoamérica (Martínez \& Javiera, 2020).

\section{Efectos negativos de la pandemia en la educación}

Es evidente que, a nivel mundial, los efectos de la pandemia por COVID-19 han limitado el desarrollo social, económico, político y especialmente el educativo (Grupo Banco Mundial, 2020). Producto de ello se ha generado:

- Deterioro de los resultados educativos

- Aumento en la pobreza de aprendizaje

- Aumento en la pobreza en general (debido a la deserción escolar)

- Aumento en la inequidad

- Aumento en el malestar social

En el área educativa, los factores mencionados han influenciado de manera negativa en la adquisición de los conocimientos, en el desarrollo de los procesos lectores; pues, el deterioro de los resultados de aprendizaje y la baja calidad de la enseñanza desembocó en un pobre aprendizaje, generando preocupación en las autoridades educativas y docentes, inquietud en los padres de familia, desesperación y estrés en los estudiantes, siendo motivo de análisis y reflexión de las entidades educativas y de los gobiernos de turno (Altamirano et al., 2021).

\section{Metodología}

La presente investigación es de tipo descriptiva, con un enfoque mixto, se llevó a cabo a través de la aplicación de encuestas que se valoraron posteriormente de forma estadística con la ayuda del programa estadístico Minitab, para la posterior descripción cualitativa de los resultados y variables propuestas.

Es de tipo correlacional ya que se establece la relación entre la lectoescritura y su enseñanza en entornos virtuales a través de la correlación de Spearman, además esde tipo bibliográfico y de campo ya que se sustenta mediante la revisión de diferentes fuentes bibliográficas que permiten la profundización teórica de las variables y la información se obtuvo directamente de los involucrados en el proceso educativo.

Los datos se recogieron a través de encuestas aplicadas a 50 representantes de niños de entre 6 y 8 años de diferentes instituciones educativas de la ciudad de Ambato, dado que 
nos podían brindar una perspectiva clara del proceso de aprendizaje que llevan a cabo sus representados. A partir de la información obtenida se desarrolló el análisis descriptivo de los aspectos relacionados con la enseñanza virtual de la lectoescritura y su consolidación durante la emergencia sanitaria.

\section{Resultados}

Tabla 5

Resultados encuesta Lectoescritura

\begin{tabular}{|c|c|c|c|c|c|c|c|c|c|c|}
\hline \multirow[b]{2}{*}{ Preguntas } & \multicolumn{10}{|c|}{ Frecuencias } \\
\hline & \multicolumn{2}{|c|}{ Siempre } & \multicolumn{2}{|c|}{$\begin{array}{c}\text { Casi } \\
\text { Siempre }\end{array}$} & \multicolumn{2}{|c|}{ Regularmente } & \multicolumn{2}{|c|}{$\begin{array}{c}\text { Casi } \\
\text { nunca }\end{array}$} & \multicolumn{2}{|c|}{ Nunca } \\
\hline y diferencias entre letras & $24 \%$ & 12 & $52 \%$ & 26 & $14 \%$ & 7 & $0 \%$ & 0 & $10 \%$ & 5 \\
\hline $\begin{array}{l}\text { Mi representado maneja de manera } \\
\text { precisa el lápiz, borrador y regla }\end{array}$ & $36 \%$ & 18 & $34 \%$ & 17 & $20 \%$ & 10 & $2 \%$ & 1 & $8 \%$ & 4 \\
\hline $\begin{array}{l}\text { Mi representado maneja la fidelidad en } \\
\text { un texto a la hora de escribir (NO } \\
\text { omite, sustituye, aumenta o invierte } \\
\text { letras) }\end{array}$ & $38 \%$ & 19 & $10 \%$ & 10 & $18 \%$ & 18 & $6 \%$ & 3 & $0 \%$ & 0 \\
\hline $\begin{array}{l}\text { Mi representado utiliza un solo tipo de } \\
\text { letra en sus escritos }\end{array}$ & $40 \%$ & 20 & $32 \%$ & 16 & $22 \%$ & 11 & $0 \%$ & 0 & $6 \%$ & 3 \\
\hline $\begin{array}{l}\text { Los escritos de mi representado tienen } \\
\text { una letra legible y ordenada }\end{array}$ & $38 \%$ & 19 & $34 \%$ & 17 & $22 \%$ & 11 & $2 \%$ & 1 & $4 \%$ & 2 \\
\hline $\begin{array}{l}\text { Mi representado lee con voz } \\
\text { audible (se escucha su lectura) }\end{array}$ & $44 \%$ & 22 & $30 \%$ & 15 & $16 \%$ & 8 & $4 \%$ & 2 & $6 \%$ & 3 \\
\hline $\begin{array}{l}\text { Mi representado lee con pronunciación } \\
\text { adecuada }\end{array}$ & $34 \%$ & 17 & $44 \%$ & 22 & $18 \%$ & 9 & $2 \%$ & 1 & $2 \%$ & 1 \\
\hline $\begin{array}{l}\text { Mi representado lee textos de forma } \\
\text { fluida }\end{array}$ & $62 \%$ & 31 & $6 \%$ & 3 & $32 \%$ & 16 & $0 \%$ & 0 & $0 \%$ & 0 \\
\hline $\begin{array}{l}\text { Mi representado comprende lecturas } \\
\text { leídas por otros }\end{array}$ & $40 \%$ & 20 & $42 \%$ & 21 & $16 \%$ & 8 & $2 \%$ & 1 & $0 \%$ & 0 \\
\hline $\begin{array}{l}\text { Mi representado relaciona textos } \\
\text { escritos con imágenes }\end{array}$ & $50 \%$ & 25 & $30 \%$ & 15 & $10 \%$ & 5 & $0 \%$ & 0 & $10 \%$ & 5 \\
\hline
\end{tabular}

Fuente: Encuesta Enseñanza virtual de la lectoescritura

Luego de la aplicación del instrumento a los representantes de los 50 niños entre 6 y 8 
años, la tabla refleja el resumen de las preguntas relacionadas con la lectoescritura, misma que denota mayor porcentaje de respuesta en la frecuencia siempre, evidenciando un adecuado aprendizaje de estas competencias.

\section{Figura 1}

Pregunta 3: Mi representado maneja la fidelidad en un texto a la hora de escribir (NO omite, sustituye, aumenta o invierte letras)

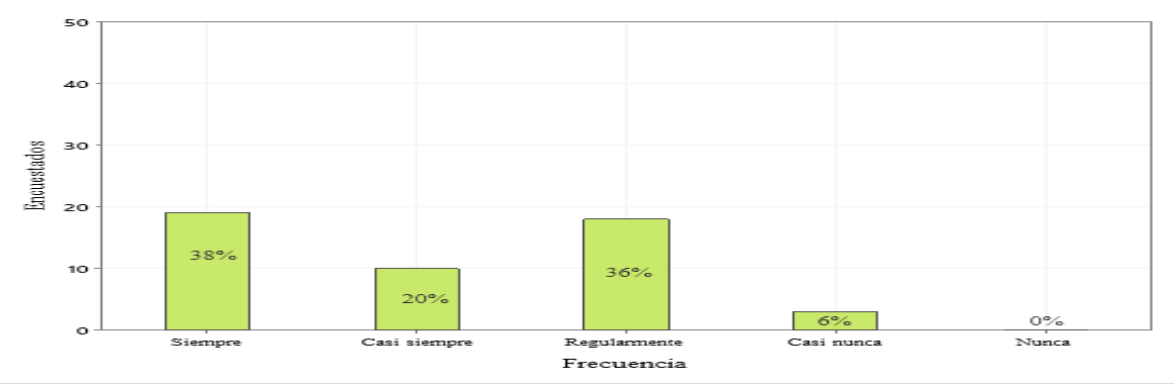

Fuente: Estudio de contextos de Unidades Educativas de la ciudad de Ambato

Luego de la aplicación del instrumento en la pregunta mi representado maneja la fidelidad en un texto a la hora de escribir, los representantes de los 50 niños entre 6 y 8 años respondieron que $38 \%$ de los estudiantes, que corresponde a 18 personas, siempre maneja la fidelidad en un texto a la hora de escribir, el $20 \%$ de los estudiantes, correspondiente a 10 personas lo realizan casi siempre, e $36 \%$ de los estudiantes, que corresponde a 17 personas que realizan frecuentemente, el $6 \%$ de los estudiantes que corresponde a 3 personas, casi nunca logran manejar la fidelidad de los textos, y el $0 \%$ está en nunca, por lo que podemos analizar que más del $50 \%$ de los estudiantes en la educación virtual si han logrado mantener la fidelidad en un texto

\section{Figura 2}

Pregunta 9: Mi representado comprende lecturas leídas por otros

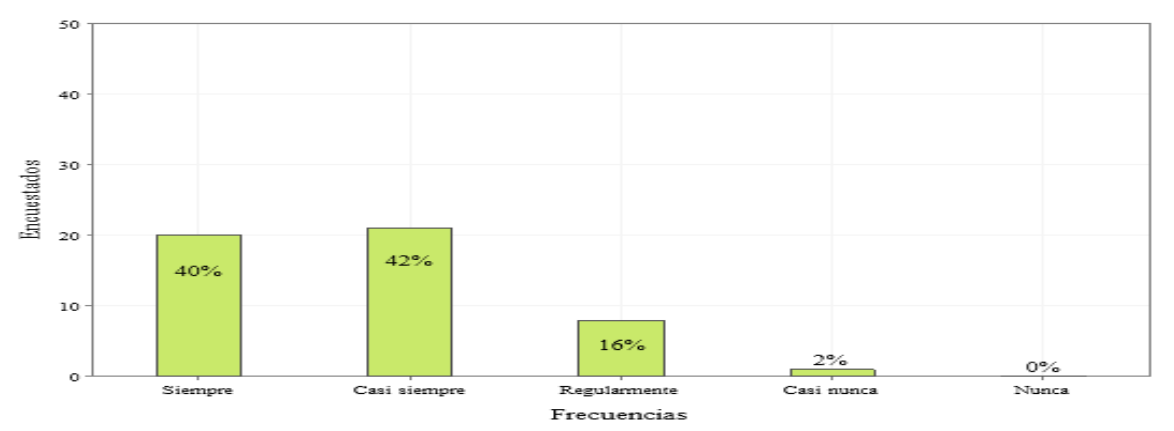

Fuente: Estudio de contextos de Unidades Educativas de la ciudad de Ambato

Dentro del análisis de las encuestas en la pregunta mi representado comprende lecturas leídas por otros, se evidencia que un $42 \%$ de la población, correspondiente a 21 representantes, consideran que los niños comprenden casi siempre textos leídos por otros, 
un $40 \%$, mismos que corresponden a 20 encuestados, lo hacen siempre. Por otro lado, se refleja que un $18 \%$, correspondiente a 8 personas, comprende lecturas en esta modalidad de forma regular, finalmente un $2 \%$, que corresponde a 1 encuestado lo hace casi nunca y el $0 \%$ corresponde a la frecuencia nunca. Estos datos nos permiten inferir que la mayor parte de la población logra comprender un texto cuando es leído por otra persona.

\section{Tabla 6}

\section{Resultados encuestas Enseñanza Virtual}

\begin{tabular}{|c|c|c|c|c|c|c|c|c|c|c|}
\hline \multirow[b]{2}{*}{ Preguntas } & \multicolumn{10}{|c|}{ Frecuencias } \\
\hline & \multicolumn{2}{|c|}{ Siempre } & \multicolumn{2}{|c|}{$\begin{array}{c}\text { Casi } \\
\text { Siempre }\end{array}$} & \multicolumn{2}{|c|}{ Regularmente } & \multicolumn{2}{|c|}{$\begin{array}{c}\text { Casi } \\
\text { nunca }\end{array}$} & \multicolumn{2}{|c|}{ Nunca } \\
\hline $\begin{array}{l}\text { necesario sobre tecnología para } \\
\text { realizar las clases virtuales }\end{array}$ & $26 \%$ & 13 & $48 \%$ & 24 & $14 \%$ & 7 & $0 \%$ & 0 & $12 \%$ & \\
\hline $\begin{array}{l}\text { El/la docente es capaz de manejar } \\
\text { archivos multimedia para la enseñanza } \\
\text { de la lectoescritura }\end{array}$ & $34 \%$ & 17 & $42 \%$ & 21 & $14 \%$ & 7 & $2 \%$ & 1 & $8 \%$ & 4 \\
\hline $\begin{array}{l}\text { El/la docente aplica estrategias de } \\
\text { interacción mediante tecnologías } \\
\text { digitales }\end{array}$ & $38 \%$ & 19 & $34 \%$ & 17 & $24 \%$ & 12 & $2 \%$ & 1 & $2 \%$ & 1 \\
\hline $\begin{array}{l}\text { El/la docente proporciona suficiente } \\
\text { material de apoyo para aplicarlo como } \\
\text { refuerzo en casa }\end{array}$ & $36 \%$ & 18 & $36 \%$ & 18 & $26 \%$ & 13 & $2 \%$ & 1 & $0 \%$ & 0 \\
\hline $\begin{array}{l}\text { El/la docente genera espacios de } \\
\text { participación activa para los } \\
\text { estudiantes durante las clases virtuales }\end{array}$ & $34 \%$ & 17 & $44 \%$ & 22 & $18 \%$ & 9 & $2 \%$ & 1 & $2 \%$ & 1 \\
\hline $\begin{array}{l}\text { El/la docente realiza una adecuada } \\
\text { evaluación de la adquisición de } \\
\text { competencias de lectoescritura }\end{array}$ & $40 \%$ & 20 & $44 \%$ & 22 & $12 \%$ & 6 & $2 \%$ & 1 & $2 \%$ & 1 \\
\hline $\begin{array}{l}\text { El/la docente brinda una adecuada } \\
\text { retroalimentación durante el proceso de } \\
\text { enseñanza y aprendizaje de la } \\
\text { lectoescritura }\end{array}$ & $38 \%$ & 19 & $46 \%$ & 23 & $14 \%$ & 7 & $2 \%$ & 1 & $0 \%$ & 0 \\
\hline $\begin{array}{l}\text { El/la docente utiliza un espacio digital } \\
\text { seguro para el desarrollo del proceso de } \\
\text { aprendizaje }\end{array}$ & $56 \%$ & 28 & $34 \%$ & 17 & $8 \%$ & 4 & $2 \%$ & 1 & $0 \%$ & 0 \\
\hline $\begin{array}{l}\text { El/la docente es capaz de solucionar } \\
\text { problemas técnicos que se generan en } \\
\text { entornos virtuales de aprendizaje }\end{array}$ & $40 \%$ & 20 & $42 \%$ & 21 & $14 \%$ & 7 & $0 \%$ & 0 & $4 \%$ & 2 \\
\hline $\begin{array}{l}\text { El/la docente utiliza herramientas } \\
\text { tecnológicas innovadoras para impartir } \\
\text { la enseñanza del proceso de } \\
\text { lectoescritura }\end{array}$ & $32 \%$ & 16 & $40 \%$ & 20 & $22 \%$ & 11 & $4 \%$ & 2 & $2 \%$ & 1 \\
\hline
\end{tabular}

Fuente: Encuesta Enseñanza virtual de la lectoescritura 
La presente tabla resume el resultado de las respuestas del instrumento relacionadas con los ítems de enseñanza virtual, misma que refleja un mayor porcentaje de respuesta en la frecuencia casi siempre. Esto demuestra que los representantes encuestados consideran que los docentes usan de manera satisfactoria las herramientas tecnológicas durante las clases virtuales.

\section{Figura 3}

Pregunta 2: El/la docente es capaz de manejar archivos multimedia para la enseñanza

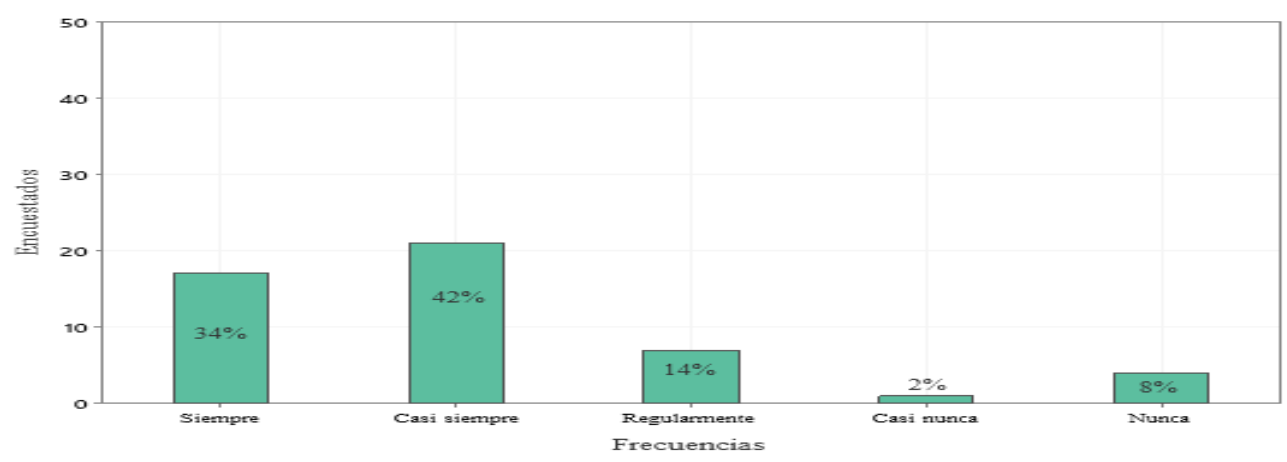

Fuente: Estudio de contextos de Unidades Educativas de la ciudad de Ambato

La grafica evidencia que un $42 \%$ de los encuestados, correspondiente a 21 personas de la población, afirman que los docentes casi siempre manejan archivos multimedia dentro de las aulas virtuales para la enseñanza de la lectoescritura, un 34\% de la población, que corresponde a 17 personas manifiesta que los docentes siempre manejan archivos multimedia, un $14 \%$ de ellas correspondientes a 7 personas, afirman que los maestros manejan de manera regular este tipo de archivos. Por otro lado, el $8 \%$, que corresponde a 4 personas nunca manejan esta condición y finalmente el $2 \%$ correspondiente a 1 persona casi nunca lo realiza. Se puede analizar que más del 50\% de los docentes manejan archivos multimedia para la enseñanza virtual de la lectoescritura.

\section{Figura 4}

Pregunta 10: El/la docente utiliza herramientas tecnológicas innovadoras para impartir la enseñanza del proceso de lectoescritura

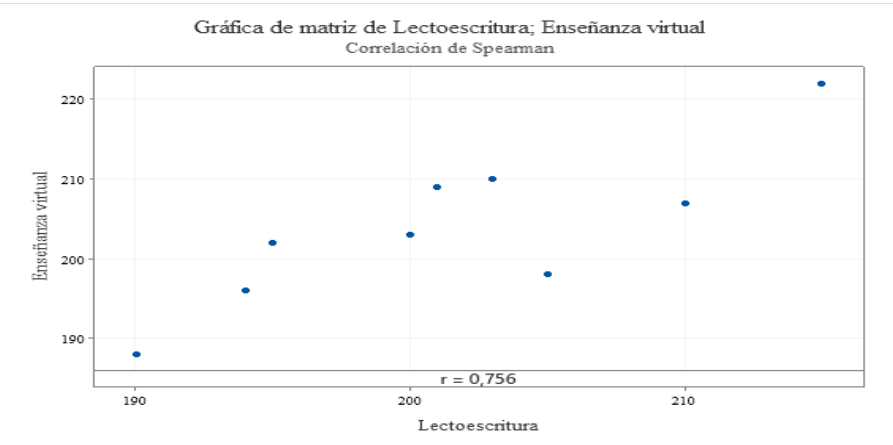

Fuente: Estudio de contextos de Unidades Educativas de la ciudad de Ambato 
Dentro de la población encuestada se identificó que un 40\% de esta, correspondiente a 20 personas, considera que casi siempre los maestros hacen uso de herramientas tecnológicas innovadoras para la enseñanza de la lectoescritura, un $32 \%$ que corresponde a 16 personas, manifiesta que lo hacen siempre. Por otro lado, un $22 \%$ de la población correspondiente a 11 personas, consideran que los maestros hacen uso de estas herramientas regularmente, finalmente los resultados con porcentaje más bajos de $4 \%$ correspondiente a 2 personas y de $2 \%$ que corresponde a 1 persona asumen que los docentes casi nunca y nunca, respectivamente, manejan este tipo de herramientas. Lo que nos deja inferir que más del $70 \%$ de la población identificó que los maestros hacen uso de herramientas tecnológicas innovadoras para la enseñanza de la lectoescritura.

Figura 5

\section{Correlación Enseñanza Virtual y Lectoescritura}

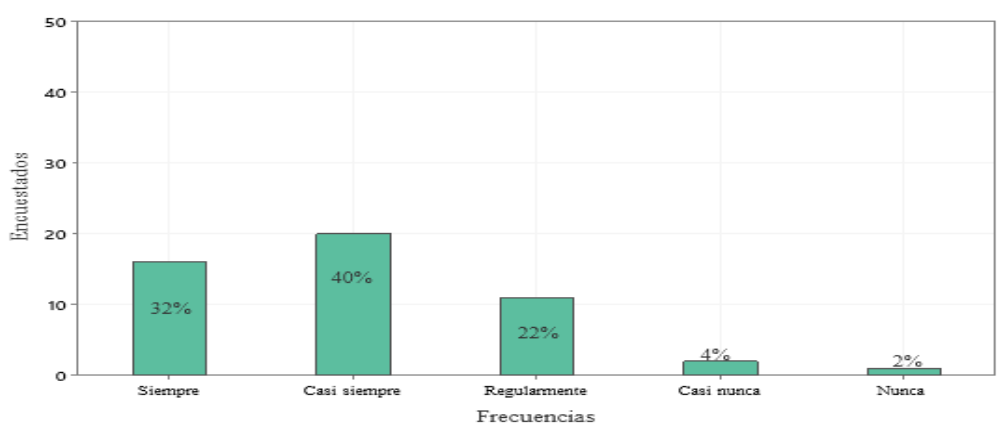

Fuente: Estudio de contextos de Unidades Educativas de la ciudad de Ambato

Finalmente, luego del análisis estadístico y de la aplicación del método de correlación de Spearman la presente grafica demuestra una correlación positiva considerable de 0,75 , lo que evidencia que el manejo adecuado o inadecuado de los recursos tecnológicos por parte de los docentes, durante las clases virtuales, repercute en los resultados de aprendizaje de la lectoescritura para que estos se consoliden de forma positiva o negativa en los niñosde entre 6 y 8 años.

\section{Conclusiones}

- En el presente estudio se consideraron las variables relacionadas con los procesos de enseñanza de la lectoescritura dentro de entornos virtuales de aprendizaje, lo cual demuestra que la aplicación de estrategias metodológicas adaptadas a la nueva modalidad de estudio, inciden de manera significativa en los resultados de aprendizaje y consecuentemente en el desempeño académico de los niños en edades comprendidas entre 6 a 8 años.

- La edad de los estudiantes motivo de la investigación es propicia para que los docentes apliquen estrategias metodológicas interactivas y motivacionales 
apoyadas en plataformas educativas, diseñadas para estimular el desarrollo de habilidades de discriminación, comparación y relación. De este modo se reafirman los procesos de percepción auditiva, visual y viso motriz, para habilitar la lectura y escritura desde edades tempranas.

- Sin embargo, se debe considerar que, para optimizar la calidad de los aprendizajes a través de la virtualidad, los docentes deben capacitarse en el uso de la tecnología y manejar con habilidad y destreza los medios digitales, lo que facilita la aplicación de los procesos de enseñanza intraulicos.

- Así también, es necesario identificar las dificultades que presentan los estudiantes en los procesos de lectura y escritura para proceder a la implementación de estrategias tecnológicas en base a los requerimientos estudiantiles y de esta manera generar aprendizajes significativos.

- Finalmente, la ejecución del presente estudio permitió valorar la necesidad de adaptar a los procesos metodológicos para el aprendizaje de la lectoescritura, herramientas tecnológicas virtuales en donde se propongan actividades sincrónicas yasincrónicas, considerando que la adquisición del conocimiento es gradual y que los nuevos contextos educativos deben contar con escenarios interactivos de enseñanza y aprendizaje.

\section{Referencias bibliográficas}

Aguilar Gordón, F. (2020). Del aprendizaje en escenarios presenciales al aprendizaje virtual en tiempos de pandemia. Estudios pedagógicos (Valdivia), 213-223.

Altamirano P, H. R., Cadena P, V. J., \& Arias V, B. E. (2021). Educación virtual y su impacto socio- económico en los estudiantes y docentes de una unidad educativa. Explorador Digital, 5(3), 85-109. https://doi.org/10.33262/exploradordigital.v5i3.1771

Aparicio Gómez, O., \& Ostos Ortiz, O. (2021). Pedagogías emergentes en ambientes virtuales de aprendizaje. Revista Internacional De Pedagogía E Innovación Educativa, 11-36.

Cabezas, L. E., Rodríguez Durán, M. E., \& Moyota Amaguaya, P. P. (2021). Conectividad y el uso de herramientas digitales en el aprendizaje del idioma inglés en estudiantes universitarios en tiempos del covid-19. ConcienciaDigital, 4(1.1), 307-326. https://doi.org/10.33262/concienciadigital.v4i1.1.1565

Cediilo, A., \& Cevallos, A. (2020). La educación virtual en el proceso del aprestamiento de la lectoescritura. Bachelor's thesis, UNIVERSIDAD DE GUAYAQUIL. Facultad de Filosofía, Letras Y Ciencias De La Educación. 
Crisol, E., Herrera, E., \& Montes, R. (2020). Educación virtual para todos: una revisión sistemática. Education in the knowledge society (EKS), 15.

Erazo Rodríguez, M. E., Calderón Cruz, F. A., Murillo Naranjo, M. E., \& Ávalos Torres, M. B. (2020). Educación interactiva: estrategia pedagógica para resignificar la identidad cultural y comprensión lectora de leyendas Riobambeñas. Ciencia Digital, 4(4), 44-64. https://doi.org/10.33262/cienciadigital.v4i4.1421

Falcon, J. (17 de noviembre de 2020). blog.bosquedefantasias.com. Obtenido de https://blog.bosquedefantasias.com/noticias/que-es-metodo-alfabeticolectoescritura.

García Guavita, S. E., \& Pito Bolaños, O. L. (2019). Descripción de las prácticas pedagógicas empleadas por los maestros de la Institución Educativa Técnica Agroindustrial" Leopoldo García" de Palocabildo-Tolima, para la iniciación de los procesos de lectura y escritura en el grado transición.

Guerrero Ávila, Z. E., Guevara Flores, L. V., \& Barrios Palacios, Y. D. (2020). Aporte metodológico de la educación física, fortaleciendo la lectoescritura en los estudiantes de segundo EGB. Conciencia Digital, 3(4), 139-145. https://doi.org/10.33262/concienciadigital.v3i4.1437

Grupo Banco Mundial. (2020). Covid-19: impacto en la educación y respuestas de política pública.

Huaraca Caisaguano, P. S. (2020). Guía didáctica para potenciar la conciencia fonológica en el aprendizaje de la lectoescritura en el segundo grado de Educación General Básica de la unidad educativa Archidona. Roca revista científico - educacional de la provincia Granma, 125.

Martínez, A. (19 de agosto de 2021). Concepto Definición. Obtenido de https://conceptodefinicion.de/lectura/

Martínez, K., \& Javiera Maestri, M. (12 de mayo de 2020). Entredichos. Obtenido de https://entredichos.trabajosocial.unlp.edu.ar/2020/05/12/educacion-virtual-entiempos-de-covid-19-barreras-de-accesibilidad-y-desigualdades-sociales/

Norman Acevedo, E. (2019). Nuevos lenguajes para aprendizaje virtual herramientas para los escenarios de aprendizaje. Panorama.

Pascual Lacal, M. R., Madrid, D., \& Estrada Vidal, L. I. (2018). Factores predominantes en el aprendizaje de la iniciación a la lectura. Revista mexicana de investigación educativa, 1122-1124. 
Pascual Llorens, S., \& Lozano Estívalis, M. (14 de Julio de 2020). El proceso de enseñanza aprendizaje de la lectoescritura en educación infatil.

Perez Reyes, D. (julio de 2019). El proceso de enseñanza-aprendizaje de la lectoescritura: una revisión teórica.

Pérez Rosas, A. (27 de septiembre de 2017). idi.edu.pe. Obtenido de https://idi.edu.pe/7factores-mejorar-la-comprension-lectora/

Ramos, H., Ramos, P., Peñalvo, F., \& Hernández, S. (2021). Validez de instrumento: percepción del aprendizaje virtual durante la COVID-19. Campus Virtuales, 111125.

Rodriguez Ledesma, E. Z. (2019). Contextualización de un método de lectoescritura para superar la limitada pronunciación en el lenguaje oral de los alumnos de primer grado en la Institución Educativa N 81739 del caserío de Cashorco, Distrito de Chugay, Provincia de Sánchez Carrión, Regi. Huamachuco, Peru.

Sotomayor, C. (18 de abril de 2021). Aprendizaje de la lectura en pandemia. Obtenido de https://www.elmostrador.cl/noticias/opinion/2021/04/18/aprendizaje-de-lalectura- en-pandemia/ 
El artículo que se publica es de exclusiva responsabilidad de los autores y no necesariamente reflejan el pensamiento de la Revista Conciencia Digital.

\section{Ciencia}

El artículo queda en propiedad de la revista y, por tanto, su publicación parcial y/o total en otro medio tiene que ser autorizado por el director de la Revista Conciencia Digital.
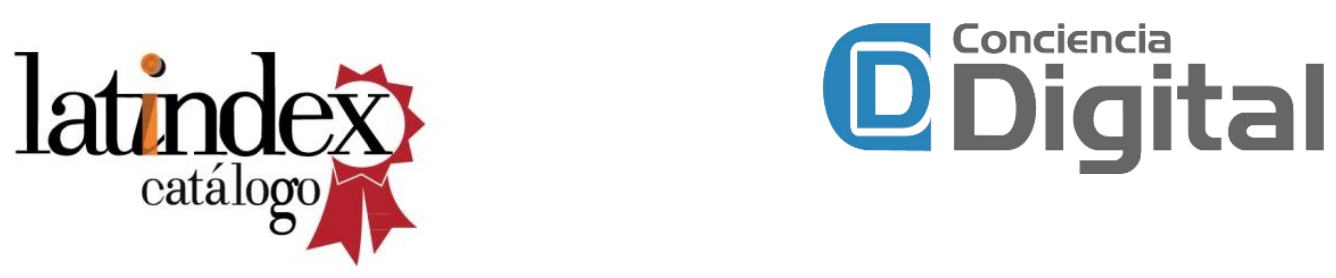

Indexaciones

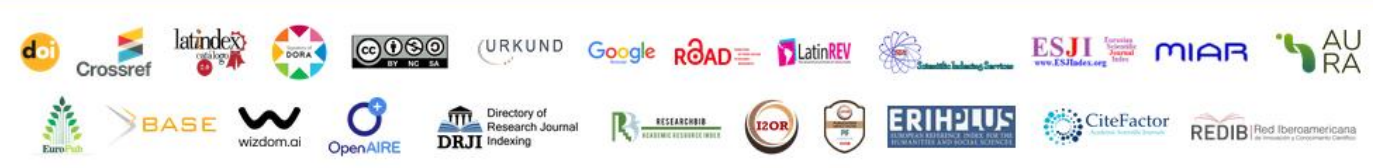

